\title{
An updated assessment of Symbiodinium spp. that associate with common scleractinian corals from Moorea (French Polynesia) reveals high diversity among background symbionts and a novel finding of clade $B$.
}

Héloïse Rouzé Corresp., ${ }^{1}$, Gaël J Lecellier ${ }^{1,2}{ }^{2}$, Denis Saulnier ${ }^{3}$, Serge Planes ${ }^{1}$, Yannick Gueguen ${ }^{4}$, Herman H Wirshing ${ }^{5}$, Véronique Berteaux-Lecellier ${ }^{1}$

1 PSL CRIOBE USR3278 CNRS-EPHE-UPVD, Labex CORAIL, BP 1013 Papetoai, 98729 Moorea, French Polynesia

2 Université de Paris Saclay, Departement de Biologie _ Versailles-Saint Quentin, 55 Avenue de Paris, 78035 Versailles Cedex, France

3 UMR241 EIO Ifremer-ILM-IRD-UPF, Labex CORAIL, BP 49, 98719 Taravao, French Polynesia

4 UMR5244 IHPE, CNRS-Ifremer-UM-UPVD, Université de Montpellier, 34095 Montpellier, France

5 Department of Invertebrate Zoology, Smithsonian National Museum of Natural History, Washington DC, USA

Corresponding Author: Héloïse Rouzé

Email address: heloise.rouze@gmail.com

The adaptative bleaching hypothesis $(\mathrm{ABH})$ states that depending on the symbiotic flexibility of coral hosts (i.e., the ability of corals to "switch" or "shuffle" their algal symbionts), coral bleaching can lead to a change in the composition of their associated Symbiodinium community, and, thus, contribute to the coral's overall survival. In order to determine the flexibility of corals, molecular tools are required to provide accurate species delineations, and to detect low levels of coral-associated Symbiodinium. Here, we used highly sensitive quantitative (real-time) PCR (qPCR) technology to analyse five common coral species from Moorea (French Polynesia), previously screened using only traditional molecular methods, to assess the presence of low-abundance (background) Symbiodinium spp.. Similar to other studies, each coral species exhibited a strong specificity to a particular clade, irrespective of the environment. In addition, however, each of the five species harboured at least one additional Symbiodinium clade, among clades A-D, at background levels. Unexpectedly, and for the first time in French Polynesia, clade B was detected as a coral symbiont. These results increase the number of known coral-Symbiodinium associations from corals found in French Polynesia, and likely indicate an underestimation of the ability of the corals in this region to associate with and/or "shuffle" different Symbiodinium clades. Altogether our data suggest that corals from French Polynesia may favor a trade-off between optimizing symbioses with a specific Symbiodinium clade(s), maintaining associations with particular background clades that may play a role in the ability of corals to respond to environmental change. 
1 An updated assessment of Symbiodinium spp. that associate with common scleractinian

2 corals from Moorea (French Polynesia) reveals high diversity among background

3 symbionts and a novel finding of clade $B$.

4 H. Rouzé ${ }^{1, \#}$, G. Lecellier ${ }^{1,2, * \#}$, D. Saulnier ${ }^{3, \#}$, S. Planes ${ }^{1, \#}$, Y. Gueguen ${ }^{4, \#}$, H. H. Wirshing ${ }^{5}$, V.

5 Berteaux-Lecellier ${ }^{1, * \#}$

7 1. PSL - USR3278 CRIOBE CNRS-EPHE-UPVD, BP 1013 Papetoai, 98729 Moorea, Polynésie 8 française

9 2. Université de Paris Saclay - Versailles-Saint Quentin, 55 Avenue de Paris, 78035 Versailles 10 Cedex, France.

11 3. UMR241 EIO Ifremer-ILM-IRD-UPF, BP 49, 98719 Taravao, Polynésie française

12 4. UMR5244 IHPE, CNRS-Ifremer-UM-UPVD, Université de Montpellier, 34095 Montpellier, 13 France

14 5. Department of Invertebrate Zoology, Smithsonian National Museum of Natural History, P.O.

15 Box 37012, NHB MRC-163, Washington, DC 20013-7012, USA

16 * present address : UMR250/9220 ENTROPIE IRD-CNRS-UR, 101, promenade Roger-Laroque, 17 BP A5, 1398848 Noumea cedex New Caledonia.

18 \# Laboratoire d'Excellence "CORAIL"

20 Corresponding author: Héloïse Rouzé ${ }^{1, \#}$

21 1. PSL - USR3278 CRIOBE CNRS-EPHE-UPVD, BP 1013 Papetoai, 98729 Moorea, Polynésie 22 française.

23 Email address: heloise.rouze@gmail.com 


\section{ABSTRACT}

25 The adaptative bleaching hypothesis $(\mathrm{ABH})$ states that depending on the symbiotic flexibility of 26 coral hosts (i.e., the ability of corals to "switch" or "shuffle" their algal symbionts), coral 27 bleaching can lead to a change in the composition of their associated Symbiodinium community, 28 and, thus, contribute to the coral's overall survival. In order to determine the flexibility of corals, molecular tools are required to provide accurate species delineations, and to detect low levels of coral-associated Symbiodinium. Here, we used highly sensitive quantitative (real-time) PCR (qPCR) technology to analyse five common coral species from Moorea (French Polynesia), previously screened using only traditional molecular methods, to assess the presence of lowabundance (background) Symbiodinium spp.. Similar to other studies, each coral species exhibited a strong specificity to a particular clade, irrespective of the environment. In addition, however, each of the five species harboured at least one additional Symbiodinium clade, among clades A-D, at background levels. Unexpectedly, and for the first time in French Polynesia, clade B was detected as a coral symbiont. These results increase the number of known coralSymbiodinium associations from corals found in French Polynesia, and likely indicate an underestimation of the ability of the corals in this region to associate with and/or "shuffle" different Symbiodinium clades. Altogether our data suggest that corals from French Polynesia may favor a trade-off between optimizing symbioses with a specific Symbiodinium clade(s), maintaining associations with particular background clades that may play a role in the ability of corals to respond to environmental change.

\section{INTRODUCTION}



corals and dinoflagellates in the genus Symbiodinium. Molecular phylogenetic analyses currently subdivide Symbiodinium into nine clades (A-I), each divided further into sub-clades or types based on various molecular techniques (reviewed in Pochon, Putnam \& Gates, 2014). However, corals most commonly associate with Symbiodinium in clades A-D (reviewed in Baker, 2003), and, in rare cases, with members of clades F and G (LaJeunesse et al., 2010; Putnam et al., 2012; Lee et al., 2016). Symbiodinium is assumed to provide up to $95 \%$ of the energy required for coral metabolic activities (Muscatine \& Porter, 1977; Davy, Allemand \& Weis, 2012), mostly due to their photosynthetic activity (i.e., production of carbohydrates). In return, the algae benefit by receiving a protected habitat from predation, and a source of inorganic nutrients derived from the host's metabolism. However, this symbiosis can break down, depending on the degree of stress tolerance of either partner, in response to various stressors that may include natural and/or anthropogenic sources [e.g., increasing seasurface temperatures, ocean acidification, and sedimentation; (Pandolfi et al., 2011)].

The overall fitness of a coral colony depends on the biological and functional traits of the various organisms that comprise the coral holobiont, [i.e., the coral host, its Symbiodinium assemblages (Mieog et al., 2009b), and other associated microorganisms (e.g. bacteria) (Neave et al., 2016)]. Moreover, some coral holobionts display different sensitivities to environmental conditions, which can correlate with specific biological characteristics such as morphology (van Woesik et al., 2011). For example, the massive coral Porites predominately associates with a thermally tolerant Symbiodinium, type C15 (Fitt et al., 2009; Fabricius et al., 2011), and has been

67 shown to exhibit increased resistance to environmental stressors such as temperature anomalies 68 (Penin, Vidal-Dupiol \& Adjeroud, 2012), and experience lower mortality and/or bleaching rates 
69 compared to those observed for branching corals such as Acropora and Pocillopora (Penin,

70 Vidal-Dupiol \& Adjeroud, 2012). To date, both in situ (e.g. Rowan et al., 1997; Baker, 2003;

71 Berkelmans \& van Oppen, 2006; Sampayo et al., 2008) and in vitro physiological studies (e.g.

72 Banaszak, 2000; Kinzie et al., 2001; Hennige et al., 2009) suggest that Symbiodinium species are

73 characterized by intrinsic physiological properties that enable them to be differentially suited for

74 various environmental conditions.

75

Spatial partitioning of different Symbiodinium clades may occur at micro-scales within a

single coral colony depending on solar irradiance, or among individual colonies across different depths (Rowan et al., 1997; Kemp et al. 2015). In addition, coral-Symbiodinium associations may be diverse, and can include either mono or multi-clade associations (Fabina et al., 2012; Silverstein, Correa \& Baker, 2012). Moreover, these assorted Symbiodinium assemblages have been described in different coral colonies from the same species (Cunning, Glynn \& Baker, 2013), during coral ontogeny (Abrego, van Oppen \& Willis, 2009; Little, van Oppen \& Willis, 2004), and/or in 'normal' vs. 'stressful' environmental conditions (e.g. seawater temperature anomalies; Berkelmans \& van Oppen, 2006). Symbiodinium in clade D have been identified as the predominant algal symbiont in resistant coral colonies during and after massive bleaching events, and/or, more generally, in reefs exposed to local stressors such as sedimentation and eutrophication (van Oppen et al., 2001; Ulstrup \& van Oppen, 2003; LaJeunesse et al., 2010, 2014; Cooper et al., 2011). These observations highlight the importance of coral-Symbiodinium associations with respect to thermo-tolerance (Berkelmans \& van Oppen, 2006; Stat, Carter \& Hoegh-Guldberg, 2006; LaJeunesse et al., 2009). Consequently, it has been proposed that corals with flexible associations with various Symbiodinium clades (or types), such as those resulting in

91 a range of host-Symbiodinium associations, may contain an ecological advantage in the context 
92 of environmental change, a concept that is encapsulated in the 'Adaptive Bleaching Hypothesis' 93 (ABH) (Buddemeier \& Fautin, 1993).

94 The $\mathrm{ABH}$ asserts that there is potential for rapid 'adaptation' of corals facing stressful 95 conditions by a dynamic modification of their Symbiodinium community composition either by i) 96 the acquisition of resistant Symbiodinium clades from free algae present in the environment (i.e., 97 'switching') or ii) repopulation by background pre-existing resistant Symbiodinium clades (i.e., 98 'shuffling'). Therefore, in the context of the $\mathrm{ABH}$, coral flexibility (the ability of a coral species 99 to associate with multiple Symbiodinium types) is of the utmost importance, and has led to the 100 characterization of coral hosts as either 'specialists' (associating with a specific Symbiodinium 101 clade) or 'generalists' (associating with multiple Symbiodinium clades) (Fabina et al., 2012; 102 Putnam et al., 2012; Silverstein, Correa \& Baker, 2012).

104 real-time quantitative PCR (qPCR), which is up to 1000 times more sensitive than conventional 105 methods (e.g. cloning, DGGEs, RFLP) (Mieog et al., 2007), allows for the detection of 106 background symbionts (in addition to the dominant symbionts), and provides a measurable 107 degree of host flexibility among corals (Silverstein, Correa \& Baker, 2012). As a result, some 108 studies have suggested that corals may be more flexible than previously thought (Mieog et al., 109 2007, 2009a; Silverstein, Correa \& Baker, 2012). The goal of this study was to investigate the 110 degree of flexibility in host-symbiont partnerships among particular coral species from the 111 under-explored Moorea island in French Polynesia using qPCR. Coral flexibility, considered 112 here as the ability of a coral species to associate with multiple Symbiodinium clades or types in 113 different proportions, and which represents one of the main conditions of the $\mathrm{ABH}$, was tested. 114 The presence of Symbiodinium clades A-F was quantified among five coral species, 
115 Acropora cytherea, Acropora pulchra, Pocillopora damicornis, Porites rus and Pavona cactus.

116 Although each coral species displayed a high degree of specificity to a dominant Symbiodinium

117 clade, all of the coral species hosted multiple Symbiodinium clades in low abundance, including

118 partnerships never recorded in French Polynesia.

119

120 MATERIALS \& METHODS

\section{Choice of coral species}

122

Five coral species, chosen among the most common scleractinian coral genera from the

123 Pacific: Pocillopora (P. damicornis type $\beta$ sensu Schmidt-Roach et al., 2014; Genbank

124 references KY110998-KY111024), Acropora (A. cytherea and A. pulchra), Porites (P. rus) and

125 Pavona (P. cactus), were collected from a fringing reef with a depth $0.5-2.0 \mathrm{~m}$ off Moorea island

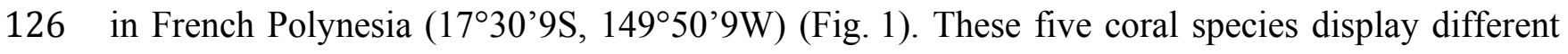

127 biological traits, and were among corals characterized as having varying resistance during severe

128 local bleaching events in 2002 and 2007 (Penin, Vidal-Dupiol \& Adjeroud, 2012). Acropora is

129 considered the "sentinel" coral genus, described as having high sensitivity to environmental

130 stressors (e.g. McClanahan et al., 2007; Penin et al., 2007; Penin, Vidal-Dupiol \& Adjeroud,

131 2012). Conversely, the genus Porites was chosen for its high resistance to stress (e.g. Kayal et

132 al., 2012; Penin, Vidal-Dupiol \& Adjeroud, 2012), living in a wide range of habitats around the

133 island (e.g., sedimentary bays). Finally, the last two genera, Pocillopora and Pavona, were

134 chosen because they are considered having intermediate degrees of sensitivity (Penin, Vidal-

135 Dupiol \& Adjeroud, 2012).

136 All of the coral species were sampled during the dry season between August and October

137 2012, P. damicornis $(\mathrm{N}=27), P$.rus $(\mathrm{N}=21)$ and A. cytherea $(\mathrm{N}=16)$ were sampled in greater 
138 proportions compared to $A$. pulchra $(\mathrm{N}=6)$ and $P$. cactus $(\mathrm{N}=7)$. Sampling was performed among

139 five contrasting fringing reefs from the lagoon of Moorea island: Mahareapa (Ma) and Vaiare

140 (Va) are exposed to anthropogenic influence, and Teavaro (Te), Linareva (Li) and Tiahura (Ti)

141 are more isolated from human activities (Nahon et al., 2013; Rouzé et al., 2015).

142

143 DNA extraction

144 Small coral fragments $\left(0.5-1 \mathrm{~cm}^{3}\right)$ were sampled at several areas from the top of each

145 coral colony, placed directly into a tube underwater, and immediately transferred at the surface

146 into a new $1.5 \mathrm{~mL}$ centrifuge tube containing $80 \%$ ethanol. All samples were stored at $-20^{\circ} \mathrm{C}$

147 until DNA extraction. Prior to extraction, all of the ethanol was discarded and the coral sample

148 gently rinsed with sterile freshwater to eliminate all traces of mucus. This allows for better

149 targeting of Symbiodinium present in the host tissues.

150 Total coral DNA (i.e., Symbiodinium, polyps, and associated micro-organisms) was

151 extracted using a CTAB-based extraction protocol adapted from Mieog et al. (Mieog et al.,

152 2009a). To increase the efficiency of DNA extraction, coral samples were incubated in $600 \mu \mathrm{L}$ of

153 extraction buffer CTAB 2\% (2\% CTAB, $1.4 \mathrm{M} \mathrm{NaCl}, 20 \mathrm{mM}$ EDTA pH 8, $100 \mathrm{mM}$ Tris-HCl

$154 \mathrm{pH} 8$ and $20 \mu \mathrm{g} / \mathrm{mL}$ proteinase $\mathrm{K}$ ). They were then exposed to 3 cryo-shock cycles (5 min in

155 liquid nitrogen following by $10 \mathrm{~min}$ at ambient temperature), and incubated at $60^{\circ} \mathrm{C}$ overnight

156 while rotating. Next, the CTAB buffer was recovered and placed into a new tube containing

$157600 \mu \mathrm{L}$ of chloroform/iso-amyl alcohol (24:1 vol/vol). The resulting solution was mixed

158 thoroughly and centrifuged for $15 \mathrm{~min}$ at $12000 \mathrm{~g}\left(4^{\circ} \mathrm{C}\right)$. The aqueous phase was then transferred

159 into a new tube and mixed with $600 \mu \mathrm{L}$ of isopropanol at $0^{\circ} \mathrm{C}$, and incubated for $20 \mathrm{~min}$ at -

$16020^{\circ} \mathrm{C}$. After a new round of centrifugation, the supernatant was discarded and the pellet rinsed 
161 with $500 \mu \mathrm{L}$ of $70 \%$ ethanol. After a final centrifugation of $10 \mathrm{~min}$ at $12000 \mathrm{~g}$, the ethanol was

162 removed and the DNA pellet air-dried before dilution in $100 \mu \mathrm{L}$ sterile water (Sigma). All DNA

163 samples were then stored at $-40^{\circ} \mathrm{C}$.

164

165

qPCR assays

166

Primer set assessment

167

Six primer sets optimized for the amplification of nuclear ribosomal 28S in Symbiodinium

168

clades A-F (Yamashita et al., 2011), and one coral-specific 18S primer set for the coral host (i.e.,

169 polyps) were used. The $18 \mathrm{~S}$ coral host primers (univPolyp-18SF: 5'-

170

ATCGATGAAGAACGCCAGCCA-3'

and

univPolyp-18SR:

171 5'CAAGAGCGCCATTTGCGTTC-3') were designed with Primer 3 (Untergasser et al., 2012)

172 from the $18 \mathrm{~S}$ rDNA sequence alignment (276 sequences) of 18 coral species that are among the

173 most abundant genera found in French Polynesia (Porites spp., Pocillopora spp., Acropora spp.,

174 Montipora spp., and Povona spp.) as well as Symbiodinium clades as negative controls.

175 The quality of the different primer sets for qPCR was confirmed using the evaluation of

176 indicators of specificity and efficiency. Firstly, the specificity of the symbiont and host primer

177 sets were verified with DNA from cultured Symbiodinium strains [available from the BURR

178 Collection (http://www.nsm.buffalo.edu/Bio/burr/); clade A: CasskB8 and Flap1, B: Pe and

179 Flap2, C: Mp, D: A001 and A014, E: RT383 and F: Sin and Pdiv44b], and with coral DNA from

180 various species (Acropora: A. pulchra, A. cytherea, A. hyacynthus; Pocillopora: P. damicornis,

181 P. verrucosa, P. meandrina; Porites rus; Pavona cactus; Montipora spp. and Fungia spp.). A

182 percentage of specificity ( $\mathrm{Sp}$ : expected with target / unexpected with non-target region) of the

183 symbiont-specific primer sets was calculated according to the formula: $\mathrm{Sp}=1-\sum\left(100 / 2^{(\mathrm{Cti}-\mathrm{Ctx})}\right)$, 
184 where Cti and Ctx are Ct obtained from a specific primer set (Cti) and from other primer sets

185 (Ctx) on the same target DNA sample. Secondly, the efficiency of the different primer sets was 186 estimated from the standard curve method based on the log-linear regression of the $\mathrm{Ct}$ values 187 with 10-fold serial dilutions of the DNA over 7 concentrations. For both Symbiodinium and the 188 coral host, the matrix for dilution was based on a series of known DNA concentrations. In order 189 to mimic multiclade associations and/or the DNA complexity, the matrix was performed by a 190 mixture of several DNA extracts equally concentrated for Symbiodinium (70 ng of each clades 191 A-F; one reference strain per clade; clade A: CasskB8, B: Pe, C: Mp, D: A001, E: RT383 and F: $192 \mathrm{Sin}$ ), and the coral host (on 1/ mixture multi-specific: $50 \mathrm{ng}$ of the ten coral species mentioned 193 above or 2/ mixture mono-specific: $50 \mathrm{ng}$ of five DNAs from the same coral species for 194 P. damicornis, P. rus or A. cytherea). Additionally, for Symbiodinium the standard curve method 195 was applied on a series of known 28S rDNA copy numbers (amplified DNA for clades A-F;

196 Supplementary method), or a series of known cell densities of clade A, C and D isolated from the 197 coral hosts (clade B was not available; Supplementary method). Percentage of efficiency (100\% 198 of efficiency indicates that the amount of PCR product doubles during each cycle) was the ratio 199 of the observed slope and the expected slope (-3.322) of the log-linear regression. In addition, the 200 standard curves of efficiency for each corresponding primer set denoted sensitivity, which 201 corresponded to the threshold of $\mathrm{Ct}$ ranges to ensure an accurate amplification (i.e., the limits of 202 the detectable log-linear range of the PCR).

203

204

Quantification of Symbiodinium in coral hosts

In order to compare Symbiodinium clades amount between different coral samples, the 206 raw number of 28 S copies of each Symbiodinium clade (from A to F) was normalized within 
207 coral hosts to evaluate the Symbiodinium densities per sample. For each coral DNA sample, a

208 value of polyp unit was estimated by the $18 \mathrm{~S}$ copy quantification using the standard curve 209 equation (Fig. S1b) in order to normalize the quantification of the Symbiodinium clades in 28S 210 copy number, or in cell number per unit of $18 \mathrm{~S}$ polyp.

212 Green. Each reaction was performed, in a final volume of $25 \mu \mathrm{L}$ containing: $12.5 \mu \mathrm{L}$ of Brillant ${ }^{\circledR}$ 213 SYBR Green Master Mix reagent, $2.5 \mu \mathrm{L}$ of both reverse and forward primers diluted at the 214 concentration of $4 \mu \mathrm{M}$, and $10 \mu \mathrm{L}$ of DNA at various concentrations for standard curve analysis 215 or at $1 \mathrm{ng} . \mu \mathrm{L}^{-1}$ for field sample analysis. The following run protocol was performed: 1 cycle of 216 pre-incubation of $10 \mathrm{~min}$ at $95^{\circ} \mathrm{C} ; 40$ cycles of amplification: $30 \mathrm{~s}$ at $95^{\circ} \mathrm{C}, 1 \mathrm{~min}$ at $60^{\circ} \mathrm{C}$ or $64^{\circ} \mathrm{C}$ 217 for Symbiodinium and coral host respectively, and 1 min at $72^{\circ} \mathrm{C}$; and a final step, for melting 218 temperature curve analysis, of $1 \mathrm{~min}$ at $95^{\circ} \mathrm{C}, 30 \mathrm{~s}$ at $60^{\circ} \mathrm{C}$ and $30 \mathrm{~s}$ at $95^{\circ} \mathrm{C}$. Each sample was 219 analysed twice on the same plate, as one technical replicate, and averaged when the variation 220 between both $\mathrm{Ct}$ values was not exceeding 1 (if not, samples were re-processed until $\Delta \mathrm{Ct} \leq 1$ ). An 221 interplate calibrator (i.e., positive control with known concentrations and Ct values: mixture of 222 DNA from Symbiodinium clades A-F), tested in triplicate (one technical replicate), was added to 223 each plate to calibrate $\mathrm{Ct}$ values (performed manually on the MxPro software to set the 224 fluorescent threshold to a fixed Ct value) among different plates of coral DNA samples. Positive 225 amplifications were taken into account only when both technical replicates produced $\mathrm{Ct}$ values 226 inferior to the estimated threshold ranges (i.e., limit sensitivity to ensure an accurate 227 quantification; Table S1) after correction with the interplate calibrator. In addition, all melting 228 curve analyses ensured the specificity of the amplifications (Table S1). For new partnerships 229 between Symbiodinium clade(s) and coral species, we further purified the qPCR products ( 100 
230 bp) using QiaEx II Gel Extraction Kit (Qiagen GmbH, Hilden, Germany) and sequenced in both

231 directions [GATC Biotech (Cologne, Germany)].

232

233 Statistical analysis

234 All statistical analyses were performed using R version 3.3.1 software (R Core Team, 235 2013). For each Symbiodinium clade, positively quantified in coral DNA, the symbiont/host ratio 236 (i.e., $\mathrm{S} / \mathrm{H}$ ratio) were $\log +1$ transformed for further analyses. Slopes, intercepts, and the Pearson 237 correlation coefficient $\left(\mathrm{R}^{2}\right)$ were evaluated by pairwise comparisons with Student's t-tests using 238 R package RVAideMemoire version 0.9-27 (Hervé, 2013).

239 Discriminant analysis of principal components (DAPC) on S/H ratios, available for the 5 240 coral species, was performed in R package ade4 version 1.7-4 (Dray \& Dufour, 2007) in order to 241 characterize their preferential endosymbiotic assemblages and densities. Therefore, the 242 discrimination represented by ellipses was applied through the coral species as factor.

244 RESULTS

245 Validation and optimization of qPCR assays

246 For all clade-specific primer sets, the specificity of each qPCR assay was greater than $24798 \%$ (Tables S1 and S2), and was characterized by a unique melting temperature (Table S1), 248 confirming the high accuracy of each primer set to its targeted sequence. All clade-specific 249 primers yielded a good fit linear regression with similar efficiencies close to the desired aim of $250100 \%\left(95-101 \%\right.$; Table S1), strong linear correlations $\left(\mathrm{R}^{2}>0.985\right.$; Fig. S1) between $\mathrm{Ct}$ and 251 concentrations of DNA template, and no significant differences among slopes. This indicates that 252 the increase in clade-specific Symbiodinium quantity is directly proportional to the number of 
253 amplification cycles regardless of whether the tests were performed on DNA from either

254 Symbiodinium culture strains (Table S1 and see Yamashita et al., 2011), purified PCR products

255 (Fig. S1a), or from counted Symbiodinium cells (Fig. S2). The sensitivity of the clade-specific

256 primers allowed two groups of primer sets to be distinguished. Pairwise comparisons of the

257 intercepts (Student's t-test, $\mathrm{p}<0.05$ ) between the standard regression lines of 28S amplicons (Fig.

258 S1a) showed earlier detection of the primers specific to clades $A, B, E$ and $F(i=16.36 \pm 0.39$;

259 Fig. S1a) when compared with the clade-specific primers to clades C and D ( $i=19.83 \pm 0.27$; Fig.

260 S1a). From the Symbiodinium cell extraction, clade D sensitivity was significantly different from

261 clades A and C (Student's t-tests, pairwise comparisons of slopes: D/A $P<0.005$ and $\mathrm{D} / \mathrm{C}$

$262 P=0.104$; intercepts: D/A $P<0.001$ and $\mathrm{D} / \mathrm{C} P=0.015$; Fig. S2). The threshold of $28 \mathrm{~S}$ copy

263 number estimation for each clade A-F, evaluated by the absolute quantification of Symbiodinium

264 clades, was effective under 200 copies of the gene (Table S1 and Fig. S1a).

265 Similarly, the specificity of the coral-specific primer set was confirmed with positive 266 amplifications from 10 coral species and no amplifications with Symbiodinium DNA. In addition,

267 the amplification of multi (mixture of 10 coral species) vs. mono-specific (mixture of P. rus, P.

268 damicornis or A. cytherea) mixes with the coral-specific primer set yielded a good fit linear

269 regression with similar efficiencies that were close to the desired aim of $100 \%(101 \%$; Table

$270 \mathrm{~S} 1 \mathrm{~b})$, contained strong linear correlations ( $\mathrm{R}^{2}>0.99$; Fig. S1b) between $\mathrm{Ct}$ and concentration of

271 DNA template, and demonstrated no significant differences among linear correlation slopes and

272 intercepts (Student's t-tests, pairwise comparisons among the 4 DNA mixes: $P>0.05$ ). In order

273 to consider the higher complexity of multi-partner coral DNA, we performed and used analyses

274 on multi-specific mixes of Symbiodinium and coral hosts to quantify the different Symbiodinium 275 clades in coral DNA samples. 


\section{Diversity and flexibility of dominant vs. background Symbiodinium clades}

278

279

280

281

282

283

284

285

286

287

288

289

290

291

292

293

294

295

296

297

298

Symbiodinium clades A, C and D (among the tested clades A-F) were detected at least once in association with each of the five coral species studied, except for P. cactus which was never found associated with clade A (Fig. 2). The quantification of these clades either by $28 \mathrm{~S}$ copy number or by cell density displayed similar orders of magnitudes when present, whatever the species (Fig. 2A, B). For some coral species, this represents novel associations for corals from Moorea: clade C for both Acropora species, A. cytherea and A. pulchra, clade D for P. cactus, and clades A and D for P. rus (Table 1). The corresponding $28 \mathrm{~S}$ sequences for these novel coral-Symbiodinium partnerships revealed the presence of lineages within sub-clades: A13, C15, C1, and D1 (Table 1; Supplementary datas: Fig. S3 and dataset 3). In addition, Symbiodinium clade B was detected in P. damicornis $(\mathrm{N}=2$; Fig. 2), albeit in low abundances equivalent to 26 and 183 copies of $28 \mathrm{~S}$ ( 4.25 and 6.21 in $\log +1$, respectively Fig. 2A). However, no relationship was available to estimate this clade's cell number. The presence of clade B was confirmed by a match to a sequence within the sub-clade B1 (PDAM2_Moo; Supplementary datas: Fig. S3 and dataset 3). Two slightly different profiles in temperature melting curves were obtained with clade $\mathrm{C}$ amplification for P. rus. Their sequences showed that each profile corresponded to two distinct lineages within sub-clades (Supplementary datas: Fig. S3 and dataset 3): $\mathrm{C} 1$ (Tm $\sim 82.95^{\circ} \mathrm{C}$; PRUS5_Moo and PRUS6_Moo; Supplementary datas: Fig. S3 and dataset 3) and $\mathrm{C} 15\left(\mathrm{Tm} \sim 83.5^{\circ} \mathrm{C}\right.$; PRUS3_Moo and PRUS4_Moo; Supplementary datas: Fig. S3 and dataset 3). In subsequent analyses of the Symbiodinium community composition, each clade was expressed by $28 \mathrm{~S}$ copy number per unit of coral $18 \mathrm{~S}$ in order to cover clades A-F. The $\mathrm{S} / \mathrm{H}$ ratio calculation displayed intra and inter-specific variation of the total Symbiodinium densities 
299 harbored within the host (Fig. 2A), either for a specific clade or from the total Symbiodinium 300 density (all clade(s) included).

301 The occurrence of clades A, B, C, and D led to fifteen possible theoretical patterns among 302 which nine have been observed previously, including assemblages of three clades together (Fig. 303 2): $\mathrm{ACD}$ (A. cytherea and A. pulchra), $\mathrm{BCD}$ (P. damicornis) or $\mathrm{ABC}$ (P. damicornis). However, 304 Symbiodinium patterns that include clade B as either a unique clade (B) or as an additional clade 305 (BA, BC, BD, BAD and ABCD) have never been recorded. Using the Symbiodinium densities 306 (S/H ratio) within the coral host (Fig. 2C), relative proportions were determined, and allowed for 307 their classification as either dominant $(>5 \%)$ or background clade(s) $(\leq 5 \%$; Table 2$)$. 308 Symbiodinium clade B, only detected in P. damicornis, was always characterized as background 309 regardless of the clade pattern (0.0002-0.0009\% of the Symbiodinium communities; Table 2), 310 and was systematically associated with at least clade C. All of the other three clades (A, C and D) were observed at least once as background clades, depending on the species and on the clade 312 pattern. For example, clade A was occasionally found as background in P. rus with an AC313 pattern $(0.0001 \%$ within $\mathrm{Li}-05$ and $0.002 \%$ within $\mathrm{Va}-03)$, and was frequently observed as 314 background in A. cytherea ( $<2 \%$; Table 2). Clade D was found as background in P. rus $(0.026 \%$ 315 within Va-05) or P. cactus (0.003\% within Ti-05) with a CD-pattern. Clade C was observed as a 316 background clade only once in P. damicornis with a CD-pattern $(0.04 \%$ within Li-01). In some 317 corals, different Symbiodinium clades occurred in more even proportions. For example, clades C 318 (51.07\%) and D (48.93\%) within P. damicornis (Li-02; Fig. 2C) exhibited a BCD-pattern, and 319 clades A (57.13 \%) and D (42.87 \%) showed AD-pattern within A. cytherea (Va-03; Fig. 2C).

\section{Selective coral-Symbiodinium partnerships}


323 species showed compositional differences among associated communities of Symbiodinium

324 according to clade identity and to their density in the host. The first axis (43.9\% of total

325 variance) of the DAPC opposed Symbiodnium communities characterized with higher clade D

326 density (Pearson's corelation: $P<0.001, \mathrm{t}=15.7$ ) from communities composed of higher clade

327 C (Pearson's correlation: $P<0.001, \mathrm{t}=-21.5$ ) and/or clade B (Pearson's correlation: $P=0.01$,

$328 \mathrm{t}=-2.5)$ densities. Clade $\mathrm{D}$ was strongly representative of P. damicornis Symbiodinium

329 communities (100\% of coral colonies sampled), nearly always appearing as a unique clade

$330(24 / 27=89 \%$; Fig. 2). In contrast, P. rus $(18 / 21=85.7 \%$; Fig. 2$)$ and $P$. cactus $(6 / 7=85.7 \%$;

331 Fig. 2) colonies were nearly exclusively composed of mono-clade C communities. However, one

332 P. cactus colony also associated with clade D (Fig. 2), underlying a larger range of variation in

333 the density of the associated symbiotic communities (wide size of discriminant ellipse, Fig. 3).

334 The second axis (24.9\% of total variance) of the DAPC differentiated Symbiodinium

335 communities was composed of clade A (Pearson's correlation: $P<0.001, \mathrm{t}=11.4$ ), and was

336 comprised of both Acropora species. These two species mainly associated with multi-clade

337 communitities (A. cytherea: $81 \%$ and A. pulchra: $67 \%$ ) and were distinguished by a second

338 preferential clade in addition to clade A (Figs. 2, 3): D for A. cytherea (AD and ACD patterns

$33911 / 16=68.8 \%$ ) and C for $A$. pulchra (AC and ACD patterns 4/6=66.7\%).

\section{DISCUSSION}

342 This study analyzed the Symbiodinium communities of five abundant coral species from

343 Moorea (A. cytherea, A. pulchra, P. damicornis, P. cactus and P. rus), and found Symbiodinium

344 clades A, C and D (from the six clades tested, A-F) in all of the species except for P. cactus, 
345 which was never observed in association with clade A. This is congruent with previous

346 observations that have described these three Symbiodinium clades as the primary clades

347 inhabiting scleractinian corals (van Oppen et al., 2005). In contrast, while Symbiodinium clade B

348 is commonly reported in Caribbean corals (Rowan et al., 1997; Diekmann et al., 2003; Pettay \&

349 Lajeunesse, 2007; Cunning, Silverstein \& Baker, 2015), it is rarely reported in corals from the

350 Central Pacific (e.g., LaJeunesse, 2001). This study is the first record of clade B found 351 associating with corals from French Polynesia (see previous studies by Magalon, Flot \& Baudry, 352 2007; Putnam et al., 2012). Clade B was detected exclusively as a background population in 353 P. damicornis, and genotyped as belonging to sub-clade B1. Coincidentally, among the few 354 detections of Symbiodinium clade B in Pacific corals to date (e.g. LaJeunesse, 2001; Silverstein, 355 Correa \& Baker, 2012; Parkinson, Coffroth \& Lajeunesse, 2015; Lee et al., 2016), genotype B1 356 associated with $P$. damicornis in Hawaii (LaJeunesse, 2001). In addition, clade B has also been 357 found in Moorea, but as a symbiont with the nudibranch Aeolidiella alba (Wecker, Fournier \& 358 Bosserelle, 2015).

359 The low abundances of clade B may also have come from an exogenous source (e.g., surface 360 environmental cells), and therefore may represent a non-symbiotic interaction with the host. 361 However, the strict conditioning of samples during DNA extraction (e.g., eliminating traces of 362 mucus; described in Rouzé et al., 2016), and the absence of any detection of clade B in the other 363 coral species from the same sampling site considerably reduce this hypothesis. Instead, the rarity 364 and low abundance of B1 lineages in corals from Moorea may be consistent with a previous 365 report in which a B1 type was found to opportunistically associate with Pocillopora colonies 366 following a coral cold-bleaching event (LaJeunesse et al., 2010). However, a recent study by Lee 367 et al. (2016) conducted in Republic of Korea found clade B (type B2) to commonly reside in the 
368 host tissues of Alveopora japonica. Alternatively, although Pacific corals rarely associate with

369 clade B, the function of this symbiosis may represent an, as of yet, unknown ecological niche.

370 However, given the rarity of this association, the significance of this partnership it likely to have

371 minor physiological consequences on the host's survival (e.g. sensitivity to thermal stress; Loram

372 et al., 2007).

373 The qPCR assays revealed that each of the four clades A-D could be detected at least

374 once at a background level (i.e., $\leq 5 \%$ ), a finding that is consistent with previous studies (e.g.

375 Mieog et al., 2007; Silverstein, Correa \& Baker, 2012). In addition, this study increases the

376 number of known background clades, and presents novel partnerships between corals and

377 Symbiodinium (e.g. P. rus with clades A or D). However, some coral-Symbiodinium pairs were

378 not recovered. For example, P. cactus was not found associating with clade A, and P. rus,

379 P. cactus and the Acropora spp. did not associate with clade B. This could be due to a limited

380 sampling effort among some of the corals (e.g., 6 A. pulchra sampled) rather than a selective

381 exclusion by the host or symbiont to a particular partner by cellular recognition mechanisms

382 (Silverstein, Correa \& Baker, 2012; Davy, Allemand \& Weis, 2012). While a majority of

383 background clades were only occasionally detected within some coral species (e.g., clades A and

384 D in P. rus or clade B in P. damicornis), the presence of clade A in low abundance in $A$. cytherea

385 was nearly exclusive. Consequently, the ability of corals to harbour multi-clade Symbiodinium

386 communities at background levels may be due to the environmental history of Moorea island,

387 which has experienced a variety of massive bleaching events (Penin, Vidal-Dupiol \& Adjeroud,

388 2012), and therefore represents a meaningful ecological function that could influence holobiont

389 resistance (Berkelmans \& van Oppen, 2006; Mieog et al., 2007). Indeed, background clades

390 support the potential for dynamic ecological strategies (e.g., switching vs. shuffling), as 
391 described in the $\mathrm{ABH}$, that could lead to a rapid selective mechanism of tolerant coral-

392 Symbiodinium partnerships in response to environmental change (Buddemeier \& Fautin, 1993;

393 Baker, 2003).

394 Despite the observed increase in variation among Symbiodinium clade associations for the

395 five coral species studied, each species was restricted to a particular dominant or background 396 Symbiodinium clade(s). For example, clade A was exclusively observed ( $>95 \%$; Fig. 1) in the 397 Symbiodinium assemblages associated with both Acropora species, either as a background clade 398 or as a dominant clade. Similarly, other coral species exhibited a primary association with a 399 particular clade: either exclusively, as with clade C in P. rus and clade D in P damicornis, or 400 dominant, as with clade C in P. cactus. Similar to the study by Putnam et al. (2012), which 401 investigated a wide range of fringing reefs during different seasons (i.e., dry season in this study 402 vs. April: wet season), our findings also support consistent associations of coral species to 403 particular Symbiodinium clades (LaJeunesse et al., 2008; Stat et al., 2009). Given our 404 standardized sampling method, the few exceptions of the multi-clade associations found for $P$. 405 damicornis or $P$. cactus could be preferentially attributed to a transient acquisition of 406 Symbiodinium clades (Muscatine, 1973; Yamashita et al., 2011; Lee et al. 2016), rather than 407 spatial partitioning of Symbiodinium within host colonies (e.g. Rowan \& Knowlton, 1995). 408 Overall, our findings are consistent with corals as 'specialists' (low flexibility: specific to 409 particular symbiont(s)) or 'generalists' (high flexibility: associated with various symbionts). To 410 further explore symbiont diversity in corals, similar fine-scale molecular approaches (e.g., qPCR, 411 next generation sequencing; see Barbrook, Voolstra \& Howe, 2014) should be performed on a 412 wide range of coral species throughout a large geographic range. 
414 consistent with previous reports of stable partnerships between coral hosts and subsets of 415 Symbiodinium (e.g. Thornhill et al., 2006, 2009; Suwa, Hirose \& Hidaka, 2008; Rouzé et al., 416 2016). Such symbiotic specificity can be derived from the 'winnowing' of multiple symbiont 417 types initially present in the host (Nyholm \& Mcfall-Ngai, 2004). This process occurs in many 418 mutualistic relationships (e.g. legume-zhizobial bacteria: Hirsch, Lum \& Downie, 2001; squid419 luminous bacteria: Nyholm \& Mcfall-Ngai, 2004; cnidarian-Symbiodinium: Wolfowicz et al., 420 2016), and consists of a complex series of molecular recognition interactions between the host 421 and the symbionts. It is likely that the specific partnerships observed between corals and 422 Symbiodinium are derived from various biological traits (Yost et al., 2013), as well as different 423 physiological and ecological attributes among Symbiodinium clades (Kinzie et al., 2001; 424 Berkelmans \& van Oppen, 2006; Hennige et al., 2009; Baker et al., 2013) that ultimately lead to 425 the ecological success of distinct holobionts. For example, the high resistance of Porites to a 426 variety of stressors could be explained, in part, by its stable association with Symbiodinium type 427 C15 (Putnam et al., 2012). This symbiont has been characterized as thermally tolerant 428 (LaJeunesse et al., 2003; Fitt et al., 2009) and more resilient to extreme environmental conditions 429 compared to other clade C types (LaJeunesse et al., 2003), which may have favored its ecological 430 radiation throughout the Indo-Pacific (LaJeunesse, 2005; Pochon et al., 2007). In this study, and 431 similar to a previous report by Putnam et al. (2012), P. cactus always associated with 432 Symbiodinium in clade C. However, it likely belongs to type C1 (Supplemtary datas: Fig. S3 \& 433 dataset 3; Putnam et al., 2012) which is described as thermo-sensitive (Deschaseaux et al., 2014), 434 and that could explain the lower resistance to environmental conditions of the species. Similarly, 435 the ecological sensitivity of branching corals from the genera Acropora and Pocillopora could be 
436 explained, in part, by their specialization with Symbiodinium clade A (type A1; Putnam et al., 4372012 and type A13), and clade D, type D1/D1a (Putnam et al., 2012) respectively. Indeed, while 438 both clades A and D are often linked with eco-physiological benefits for the holobiont, including 439 photo-protective and thermo-tolerance abilities, respectively, they have mainly been described in 440 stressful vs. non-stressful conditions. This duality likely corresponds to trade-offs between coral 441 host resistance and low energetic budget contributions (reviewed in Lesser, Stat \& Gates, 2013). 442 In some cases, Symbiodinium belonging to these clades have been reported as nominal 443 contributors to host metabolism [e.g., growth and reproduction (Little, van Oppen \& Willis, 444 2004; Jones \& Berkelmans, 2010) and/or nutrition (Stat, Morris \& Gates, 2008; Cantin et al., 445 2009; Baker et al., 2013)].

446 The specialization of coral hosts to particular Symbiodinium clades likely represents a 447 driver resulting in stable mutualisms, initiated from selective pressure, that enhances the benefits 448 of specific symbiosis through co-evolution (Douglas, 2008; Thornhill et al., 2014). However, this 449 specialization is contrasted with the maintenance of the horizontal transmission of symbionts in 450 the majority of coral species as well as the detection of additional clades, at trace levels, within 451 the five coral species examined. Lee et al. (2016) suggest that low abundance 'background' 452 Symbiodinium populations are not necessarily mutualistic but can reflect a transient relative 453 abundance in the surrounding environment, such as non-directional ingestion by polyps leading 454 to ephemeral symbiont shifts (LaJeunesse et al., 2009; Stat et al., 2009; Coffroth et al., 2010). 455 Nevertheless, every Symbiodinium species may not be transiently ingested. For example, 456 Symbiodinium clade F was never found in the host tissues of the five coral species examined, 457 despite clade F being detected in the surrounding environment (Supplementary data: Fig. S4), 458 and, although in a temperate environment, described as a dominant symbiont within 
459 Alveopora japonica (Lee et al., 2016). This suggests a combination of physiologically controlled

460 processes among the coral host and its background Symbiodinium communities. Therefore, two

461 opposite selection pressures may be co-occuring in the context of Moorea's reef environment

462 (which has been exposed to consecutive massive bleaching events in the past): i) the

463 optimization of a symbiosis with a specific clade(s) and/or ii) the maintenance of the ability to

464 integrate several different (but not all) clades in low abundance that could yield an overall 465 benefit to the coral holobiont.

466 Altogether, these findings emphasize the need to better understand whether those

467 Symbiodinium present in low abundance play an ecological role for the holobiont over time, and

468 to further explore the processes that may govern the maintenance of Symbiodinium in low 469 abundance in addition to the dominant symbioses that occur with particular clades.

470

471 Acknowledgments. We thank B. Espiau and M. Vairaa from CRIOBE and J. Fievet (in charge 472 of qPCR platform utilization) from IFREMER from Taravao for their advices and assistance in 473 laboratory. We are also grateful to M.A. Coffroth and her laboratory (BURR; 474 http://www.nsm.buffalo.edu/Bio/burr/) for providing us cultured Symbiodinium strains. We also 475 thank C. Bonneville who performed analyses of Symbiodinium diversity in the water column 476 during her master internship. 


\section{REFERENCES}

478

479

480

481

482

483

484

485

486

487

488

489

490

491

492

493

494

495

496

497

498

499

500

501

502

503

504

505

506

507

508

509

510

511

512

513

514

515

516

Abrego D., van Oppen MJH., Willis BL. 2009. Highly infectious symbiont dominates initial uptake in coral juveniles. Molecular Ecology 18:3518-3531.

Baker AC. 2003. Flexibility and specifictity in coral-algal symbiosis: diversity, ecology, and biogeography of Symbiodinium. Annual Review of Ecology, Evolution, and Systematics 34:661-689.

Baker DM., Andras JP., Jordán-Garza AG., Fogel ML. 2013. Nitrate competition in a coral symbiosis varies with temperature among Symbiodinium clades. The ISME journal 7:1248-51.

Banaszak T. 2000. The synthesis of mycosporine-like amino acids (MAAs) by cultured, symbiotic dinoflagellates. Journal of Experimental Marine Biology and Ecology 249:219-233.

Barbrook AC., Voolstra CR., Howe CJ. 2014. The chloroplast genome of a Symbiodinium sp. clade C3 isolate. Protist 165:1-13.

Berkelmans R., van Oppen MJH. 2006. The role of zooxanthellae in the thermal tolerance of corals: a "nugget of hope" for coral reefs in an era of climate change. Proceedings of the Royal Society B: Biological Sciences 273:2305-2312.

Buddemeier RW., Fautin DG. 1993. Coral bleaching as an adaptive mechanism. Bioscience 43:320-326.

Cantin NE., van Oppen MJH., Willis BL., Mieog JC., Negri AP. 2009. Juvenile corals can acquire more carbon from high-performance algal symbionts. Coral Reefs 28:405-414.

Coffroth MA., Poland DM., Petrou EL., Brazeau DA., Holmberg JC. 2010 Environmental symbiont acquisition may not be the Solution to warming seas for reef-building corals. PLoS ONE 5:e13258.

Cooper TF., Berkelmans R., Ulstrup KE., Weeks S., Radford B., Jones AM., Doyle J., Canto M., O'Leary RA., van Oppen MJH. 2011. Environmental factors controlling the distribution of Symbiodinium harboured by the coral Acropora millepora on the Great Barrier Reef. PLoS ONE 6:e25536.

Cunning R., Glynn PW., Baker AC. 2013. Flexible associations between Pocillopora corals and Symbiodinium limit utility of symbiosis ecology in defining species. Coral Reefs 32:795-801.

Cunning R., Silverstein RN., Baker AC. 2015. Investigating the causes and consequences of symbiont shuffling in a multi-partner reef coral symbiosis under environmental change. Proceedings of the Royal Society B 282:20141725.

Davy SK., Allemand D., Weis VM. 2012. Cell biology of cnidarian-dinoflagellate symbiosis. Microbiology and Molecular Biology Reviews 76:229-261.

Deschaseaux ESM., Beltran VH., Jones GB., Deseo MA., Swan HB., Harrison PL., Eyre BD. 2014. Comparative response of DMS and DMSP concentrations in Symbiodinium clades C1 and D1 under thermal stress. Journal of Experimental Marine Biology and Ecology 459:181-189. 
517 Diekmann O., Olsen J., Stam W., Bak R. 2003. Genetic variation within Symbiodinium clade B 518 from the coral genus Madracis in the Caribbean (Netherlands Antilles). Coral Reefs $51922: 29-33$.

520

521

522

523

524

525

526

527

528

529

530

531

532

533

534

535

536

537

538

539

540

541

542

543

544

545

546

547

548

549

550

551

552

553

554

555

556

557

Douglas AE. 2008. Conflict, cheats and the persistence of symbioses. New Phytologist 177:849-858.

Dray S., Dufour AB. 2007. The ade4 package: implementing the duality diagram for ecologists. Journal of Statistical Software 22:1-20.

Fabina NS., Putnam HM., Franklin EC., Stat M., Gates RD. 2012. Transmission mode predicts specificity and interaction patterns in coral-Symbiodinium networks. PLOS ONE 7:9.

Fabricius KE., Langdon C., Uthicke S., Humphrey C., Noonan S., De'ath G., Okazaki R., Muehllehner N., Glas MS., Lough JM. 2011. Losers and winners in coral reefs acclimatized to elevated carbon dioxide concentrations. Nature Climate Change 1:165169.

Fitt WK., Gates RD., Hoegh-Guldberg O., Bythell JC., Jatkar A., Grottoli AG., Gomez M., Fisher P., Lajuenesse TC., Pantos O., Iglesias-Prieto R., Franklin DJ., Rodrigues LJ., Torregiani JM., van Woesik R., Lesser MP. 2009. Response of two species of Indo-Pacific corals, Porites cylindrica and Stylophora pistillata, to short-term thermal stress: The host does matter in determining the tolerance of corals to bleaching. Journal of Experimental Marine Biology and Ecology 373:102-110.

Hennige SJ., Suggett DJ., Warner ME., McDougall KE., Smith DJ. 2009. Photobiology of Symbiodinium revisited : bio-physical and bio-optical signatures. Coral Reefs 28:179195.

Hervé M. RVAideMemoire: Diverse Basic Statistical and Graphical Functions. R Package Version 0.9-27. 2013. Available from: http://CRAN.Rproject.org/package=RVAideMemoire.

Hirsch AM., Lum MR., Downie JA. 2001. What makes the rhizobia-legume symbiosis so special? Plant Physiology 127:1484-1492.

Jones A., Berkelmans R. 2010. Potential costs of acclimatization to a warmer climate: growth of a reef coral with heat tolerant vs. sensitive symbiont types. PloS ONE 5:e10437.

Kayal M., Vercelloni J., Lison de Loma T., Bosserelle P., Chancerelle Y., Geoffroy S., Stievenart C., Michonneau F., Penin L., Planes S., Adjeroud M. 2012. Predator crown-of-thorns starfish (Acanthaster planci) outbreak, mass mortality of corals, and cascading effects on reef fish and benthic communities. PLoS ONE 7:e47363.

Kemp DW., Thornhill DJ., Rotjan RD., Iglesias-Prieto R., Fitt WK., Schmidt GW. 2015. Spatially distinct and regionally endemic Symbiodinium assemblages in the threatened Caribbean reef-building coral Orbicella faveolata. Coral Reefs 34:535-547.

Kinzie RA., Takayama M., Santos SR., Coffroth MA. 2001. The adaptive bleaching hypothesis : experimental tests of critical assumptions. Biological Bulletin 200:51-58.

LaJeunesse TC. 2001. Investigating the biodiversity, ecology, and phylogeny of endosymbiotic dinoflagellates in the genus Symbiodinium using the ITS region: in 

search of a" species" level marker. Journal of Phycology 37:866-880.

559

560

561

562

563

564

565

566

567

568

569

570

571

572

573

574

575

576

577

578

579

580

581

582

583

584

585

586

587

588

589

590

591

592

593

594

595

596

597

598

LaJeunesse TC., Loh WKW., Van Woesik R., Hoegh-Guldberg O., Schmidt GW., Fitt WK. 2003. Low symbiont diversity in southern Great Barrier Reef corals, relative to those of the Caribbean. Limnology and Oceanography 48:2046-2054.

LaJeunesse TC. 2005. "Species" radiations of symbiotic dinoflagellates in the Atlantic and Indo-Pacific since the Miocene- Pliocene transition. Molecular Biology and Evolution 22:570-581.

LaJeunesse TC., Bonilla HR., Warner ME., Wills M., Schmidt GW., Fitt WK. 2008. Specificity and stability in high latitude eastern Pacific coral-algal symbioses. Limnology and Oceanography 53:719-727.

LaJeunesse TC., Smith RT., Finney J., Oxenford H. 2009. Outbreak and persistence of opportunistic symbiotic dinoflagellates during the 2005 Caribbean mass coral "bleaching" event. Proceedings of the Royal Society of London B: Biological Sciences 276:4139-4148.

LaJeunesse TC., Smith R., Walther M., Pinzon J., Pettay DT., McGinley M., Aschaffenburg M., Medina-Rosas P., Cupul-Magaòa AL., Pèrez AL. 2010. Host-symbiont recombination versus natural selection in the response of coral-dinoflagellate symbioses to environmental disturbance. Proceedings of the Royal Society of London B: Biological Sciences 277:2925-2934.

LaJeunesse TC., Wham DC., Pettay DT., Parkinson JE., Keshavmurthy S., Chen CA. 2014. Ecologically differentiated stress-tolerant endosymbionts in the dinoflagellate genus Symbiodinium (Dinophyceae) clade D are different species. Phycologia 53:305-319.

Lee MJ., Jeong HJ., Jang SH., Lee SY., Kang NS., Lee KH., Kim HS., Wham DC., LaJeunesse TC. 2016. Most low-abundance "background" Symbiodinium spp. are transitory and have minimal functional significance for symbiotic corals. Microbial Ecology 71:771-783.

Lesser MP., Stat M., Gates RD. 2013. The endosymbiotic dinoflagellates (Symbiodinium sp.) of corals are parasites and mutualists. Coral Reefs 32:603-611.

Little AF., van Oppen MJH., Willis BL. 2004. Flexibility in algal endosymbioses shapes growth in reef corals. Science 304:1492-1494.

Loram JE., Boonham N., O’Toole P., Trapido-Rosenthal HG., Douglas AE. 2007. Molecular quantification of symbiotic dinoflagellate algae of the genus Symbiodinium. The Biological Bulletin 212:259-268.

Magalon H., Flot J-F., Baudry E. 2007. Molecular identification of symbiotic dinoflagellates in Pacific corals in the genus Pocillopora. Coral Reefs 26:551-558.

McClanahan TR., Teweberhan M., Uhando CA., Maina J., Mohammed MS. 2007. Effects of climate and seawater temperature variation on coral bleaching and mortality. Ecological Monographs 77:503-525.

Mieog JC., van Oppen MJH., Cantin NE., Stam WT., Olsen JL. 2007. Real-time PCR reveals a high incidence of Symbiodinium clade D at low levels in four scleractinian corals across the Great Barrier Reef: implications for symbiont shuffling. Coral Reefs 26:449-457.

Mieog JC., van Oppen MJH., Berkelmans R., Stam WT., Olsen JL. 2009a. Quantification of 
599

600

601

602

603

604

605

606

607

608

609

610

611

612

613

614

615

616

617

618

619

620

621

622

623

624

625

626

627

628

629

630

631

632

633

634

635

636

637

638

639 algal endosymbionts(Symbiodinium) in coral tissue using real-time PCR. Molecular Ecology Resources 9:74-82.

Mieog JC., Olsen JL., Berkelmans R., Bleuler-Martinez SA., Willis BL., van Oppen MJH. 2009b. The roles and interactions of symbiont, host and environment in defining coral fitness. PLOS ONE 4:e6364.

Muscatine L. 1973. Nutrition of corals biology and geology of coral reefs, p. 77-115. In 0. A. Jones and R. Endean (ed.), Biology and geology of coral reefs, vol. 2. Academic Press, New York, NY.

Muscatine L., Porter J. 1977. Reef corals: mutualistic symbioses adapted to nutrient-poor environments. Bioscience 27:454-460.

Nahon S., Richoux NB., Kolasinski J., Desmalades M., Ferrier Pages C., Lecellier G., Planes S., Berteaux Lecellier V. 2013 Spatial and temporal variations in stable carbon $(\delta(13) \mathrm{C})$ and nitrogen $(\delta(15) \mathrm{N})$ isotopic composition of symbiotic scleractinian corals. PLoS ONE 8, e81247.

Neave MJ., Rachmawati R., Xun L., Michell CT., Bourne DG., Apprill A., Voolstra CR. 2016. Differential specificity between closely related corals and abundant Endozoicomonas endosymbionts across global scales. The ISME journal:1-15.

Nyholm SV., Mcfall-Ngai MJ. 2004. The winnowing : establishing the squid-Vibrio symbiosis. Nature Reviews Microbiology 2:632-642.

Pandolfi JM., Connolly SR., Marshall DJ., Cohen AL. 2011. Projecting coral reef futures under global warming and ocean acidification. Science 333:418-422.

Parkinson JE., Coffroth MA., Lajeunesse TC. 2015. New species of clade B Symbiodinium (Dinophyceae) from the greater Caribbean belong to different functional guilds: $S$. aenigmaticum sp. nov., $S$. antillogorgium sp. nov., $S$. endomadracis sp. nov., and $S$. pseudominutum sp. nov. Journal of Phycology 51:850-858.

Penin L., Adjeroud M., Schrimm M., Lenihan HS. 2007. High spatial variability in coral bleaching around Moorea (French Polynesia): patterns across locations and water depths. Comptes Rendus Biologies 330:171-181.

Penin L., Vidal-Dupiol J., Adjeroud M. 2012. Response of coral assemblages to thermal stress: Are bleaching intensity and spatial patterns consistent between events? Environmental Monitoring and Assessment 185:5031-5042.

Pettay DT., Lajeunesse TC. 2007. Microsatellites from clade B Symbiodinium spp. specialized for Caribbean corals in the genus Madracis. Molecular Ecology Notes 7:1271-1274.

Pochon X., Garcia-Cuetos L., Baker AC., Castella E., Pawlowski J. 2007. One-year survey of a single Micronesian reef reveals extraordinarily rich diversity of Symbiodinium types in soritid foraminifera. Coral Reefs 26:867-882.

Pochon X., Putnam HM., Gates RD. 2014. Multi-gene analysis of Symbiodinium dinoflagellates: a perspective on rarity, symbiosis, and evolution. PeerJ 2:e394.

Putnam HM., Stat M., Pochon X., Gates RD. 2012. Endosymbiotic flexibility associates with environmental sensitivity in scleractinian corals. Proceedings of the Royal Society B: Biological Sciences 279:4352-4361. 
640 R Core Team (2013). R: A language and environment for statistical computing. R

641

642

643

644

645

646

647

648

649

650

651

652

653

654

655

656

657

658

659

660

661

662

663

664

665

666

667

668

669

670

671

672

673

674

675

676

677

678

679

680

Foundation for Statistical Computing, Vienna, Austria. URL http://www.Rproject.org/.

Rouzé H., Lecellier G., Langlade M., Planes S., Berteaux-Lecellier V. 2015. Fringing reefs exposed to different levels of eutrophication and sedimentation can support the same benthic communities. Marine Pollution Bulletin 92:212-221.

Rouzé H., Lecellier G., Saulnier D., Berteaux-Lecellier V. 2016. Symbiodinium clades A and D differentially predispose Acropora cytherea to disease and Vibrio spp. colonization. Ecology and Evolution 6:560-572.

Rowan R., Knowlton N., Baker A., Jara J. 1997. Landscape ecology of algal symbionts creates variation in episodes of coral bleaching. Nature 388:265-269.

Rowan R., Knowlton N. 1995. Intraspecific diversity and ecological zonation in coral-algal symbiosis. Proceedings of the National Academy of Sciences 92:2850-2853.

Sampayo EM., Ridgway T., Bongaerts P., Hoegh-Guldberg 0. 2008. Bleaching susceptibility and mortality of corals are determined by fine-scale differences in symbiont type. Proceedings of the National Academy of Sciences 105:10444-10449.

Schmidt-Roach S., Miller KJ., Lundgren P., Andreakis N. 2014. With eyes wide open: a revision of species within and closely related to the Pocillopora damicornis species complex (Scleractinia; Pocilloporidae) using morphology and genetics. Zoological Journal of the Linnean Society 170:1-33.

Silverstein RN., Correa AMS., Baker AC. 2012. Specificity is rarely absolute in coral-algal symbiosis: implications for coral response to climate change. Proceedings of the Royal Society B: Biological Sciences 279:2609-2618.

Stat M., Loh WKW., LaJeunesse TC., Hoegh-Guldberg O., Carter DA. 2009. Stability of coralendosymbiont associations during and after a thermal stress event in the southern Great Barrier Reef. Coral Reefs 28:709-713.

Stat M., Carter D., Hoegh-Guldberg 0. 2006. The evolutionary history of Symbiodinium and scleractinian hosts-symbiosis, diversity, and the effect of climate change. Perspectives in Plant Ecology, Evolution and Systematics 8:23-43.

Stat M., Morris E., Gates RD. 2008. Functional diversity in coral-dinoflagellate symbiosis. Proceedings of the National Academy of Sciences of the United States of America 105:9256-61.

Suwa R., Hirose M., Hidaka M. 2008. Seasonal fluctuation in zooxanthellar genotype composition and photophysiology in the corals Pavona divaricata and P. decussata. Marine Ecology Progress Series 361:129-137.

Thornhill DJ., LaJeunesse TC., Kemp DW., Fitt WK., Schmidt GW. 2006. Multi-year, seasonal genotypic surveys of coral-algal symbioses reveal prevalent stability or post-bleaching reversion. Marine Biology 148:711-722.

Thornhill DJ., Xiang Y., Fitt WK., Santos SR. 2009. Reef endemism, host specificity and temporal stability in populations of symbiotic dinoflagellates from two ecologically dominant Caribbean corals. PLoS ONE 4:e6262. 
681 Ulstrup KE., van Oppen MJH. 2003. Geographic and habitat partitioning of genetically

682

683

684

685

686

687

688

689

690

691

692

693

694

695

696

697

698

699

700

701

702

703

704

705

706 distinct zooxanthellae (Symbiodinium) in Acropora corals on the Great Barrier Reef. Molecular Ecology 12:3477-3484.

Untergasser A., Cutcutache I., Koressaar T., Ye J., Faircloth BC., Remm M., Rozen SG. 2012. Primer3-new capabilities and interfaces. Nucleic Acids Research 40:e115-e115.

van Oppen MJH., Palstra FP., Piquet AMT., Miller DJ. 2001. Patterns of coral-dinoflagellate associations in Acropora: significance of local availability and physiology of Symbiodinium strains and host-symbiont selectivity. Proceedings of the Royal Society of London B: Biological Sciences 268:1759-1767.

van Oppen MJH., Mieog JC., Sánchez CA., Fabricius KE. 2005. Diversity of algal endosymbionts (zooxanthellae) in octocorals: the roles of geography and host relationships. Molecular Ecology 14:2403-2417.

van Woesik R., Sakai K., Ganase A., Loya Y. 2011. Revisiting the winners and the losers a decade after coral bleaching. Marine Ecology Progress Series 434:67-76.

Wecker P., Fournier A., Bosserelle P. 2015. Dinoflagellate diversity among nudibranchs and sponges from French Polynesia: Insights into associations and transfer. Comptes Rendus Biologies 338:278-283.

Wolfowicz I., Baumgarten S., Voss PA., Hambleton EA., Voolstra CR., Hatta M., Guse A. 2016. Aiptasia sp. larvae as a model to reveal mechanisms of symbiont selection in cnidarians. Scientific Reports:srep32366.

Yamashita H., Suzuki G., Hayashibara T., Koike K. 2011. Do corals select zooxanthellae by alternative discharge? Marine Biology 158:87-100.

Yost DM., Wang L., Fan T., Chen C., Lee RW., Sogin E., Gates RD. 2013. Diversity in skeletal architecture influences biological heterogeneity and Symbiodinium habitat in corals. Zoology 116:262-269. 
707 Figure 1 Map of Moorea island (Archipelago of society, French Polynesia) and the locations of 708 the fringing reefs studied (black circles). Locations (FG) with an " $\mathrm{x}$ " indicate the sites 709 investigated previously by Putnam et al. (2012). Vaiare (Va), Teavaro (Te), Maharepa (Ma), 710 Tiahura (Ti) and Linereva (Li).

711

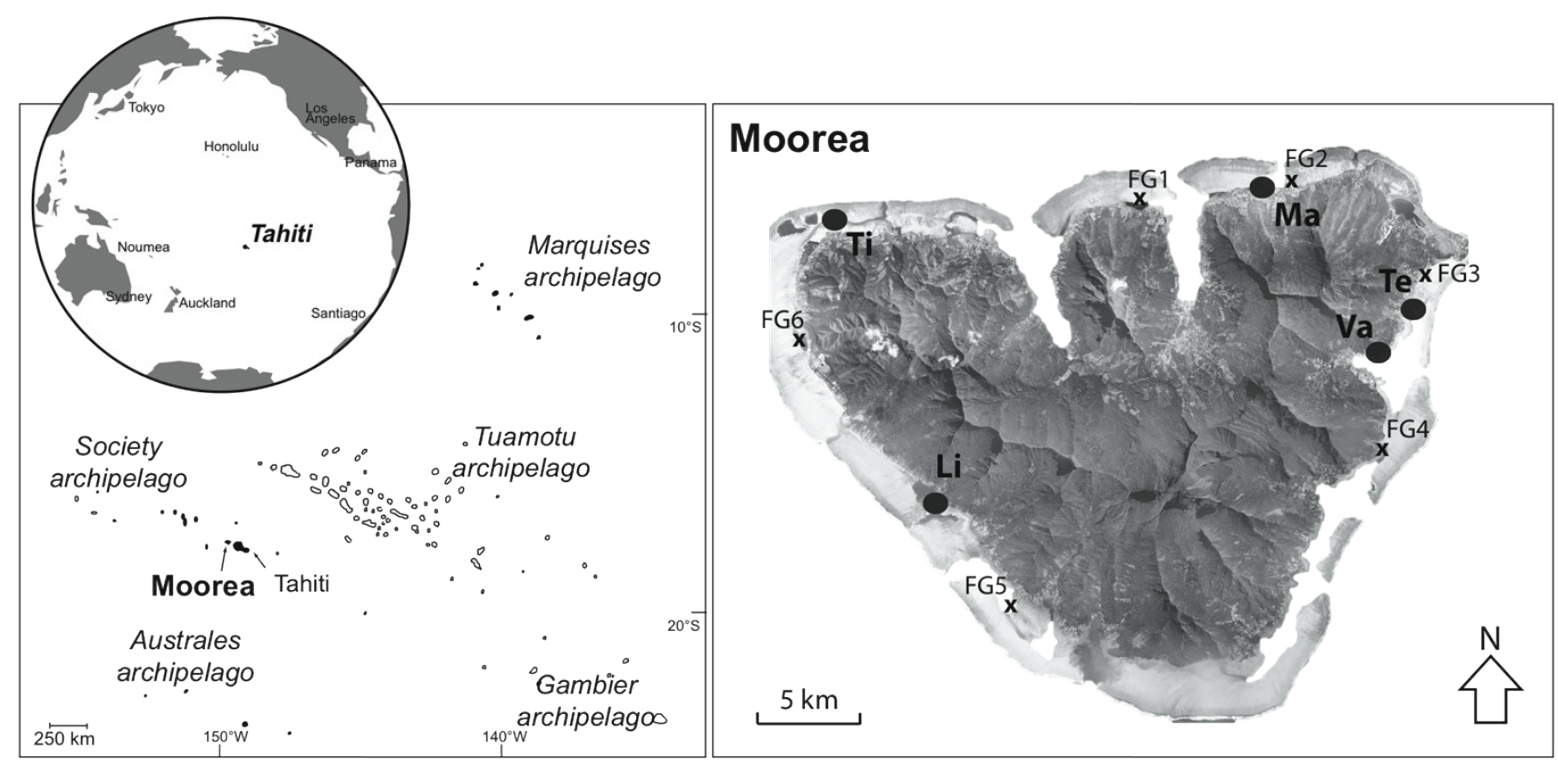


714 Figure 2 Quantitative composition of different Symbiodinium clades observed in association

715 with ACYT: A.cytherea, APUL: A. pulchra, PCAC: P. cactus, PDAM: P. damicornis and

716 PRUS: P. rus based on: (A) 28S copy number estimation (B) cell number estimation and (C)

717 clade proportions within coral hosts: the grey circles represent the presence of background clades

718 under a $5 \%$ threshold (dashed line). Coral IDs are indicated under each histogram. 


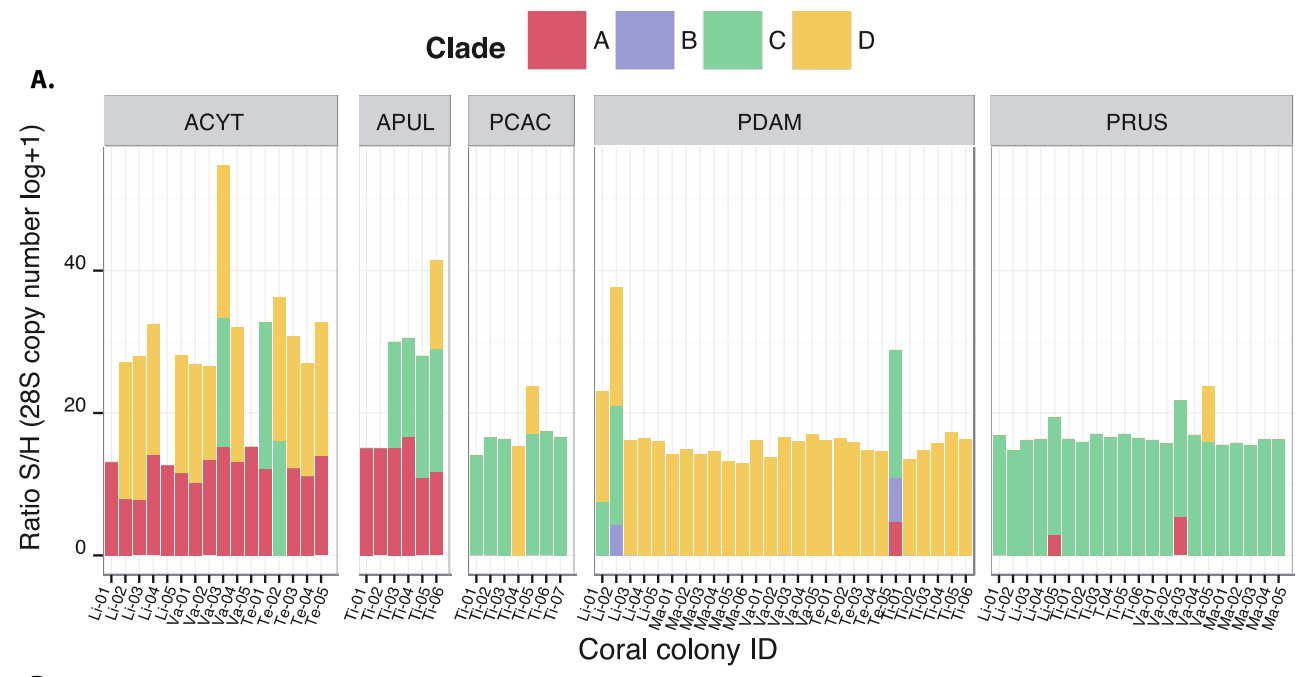

B.

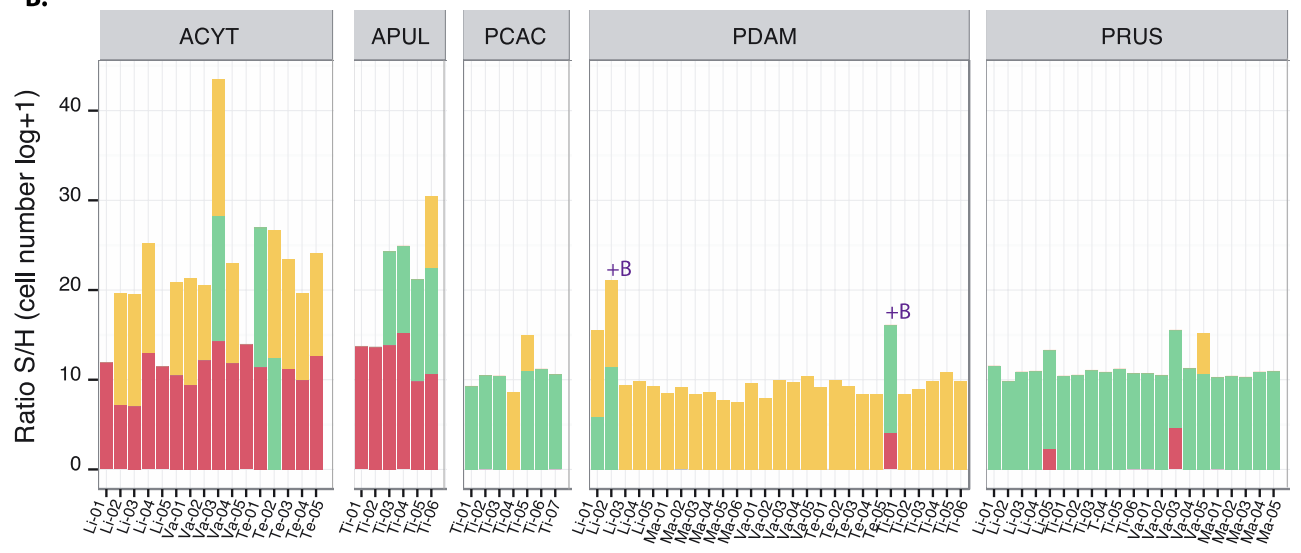

c.

Coral colony ID
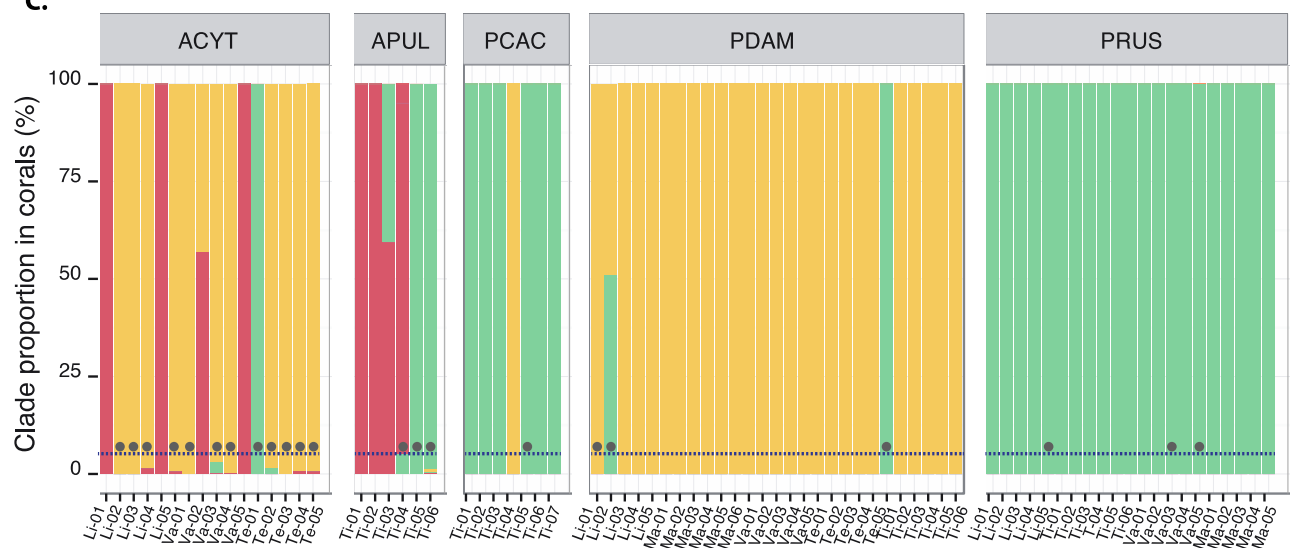

Coral colony ID 
720 Figure 3 Spatio-temporal multivariate analysis of clade A-D quantifications converted in $28 \mathrm{~S}$

721 copy number. Axis 1 and 2 of the discriminant analysis of principal component (DAPC)

722 according to the five coral species: A. cytherea, A. pulchra, P. cactus, P. damicornis and P. rus.

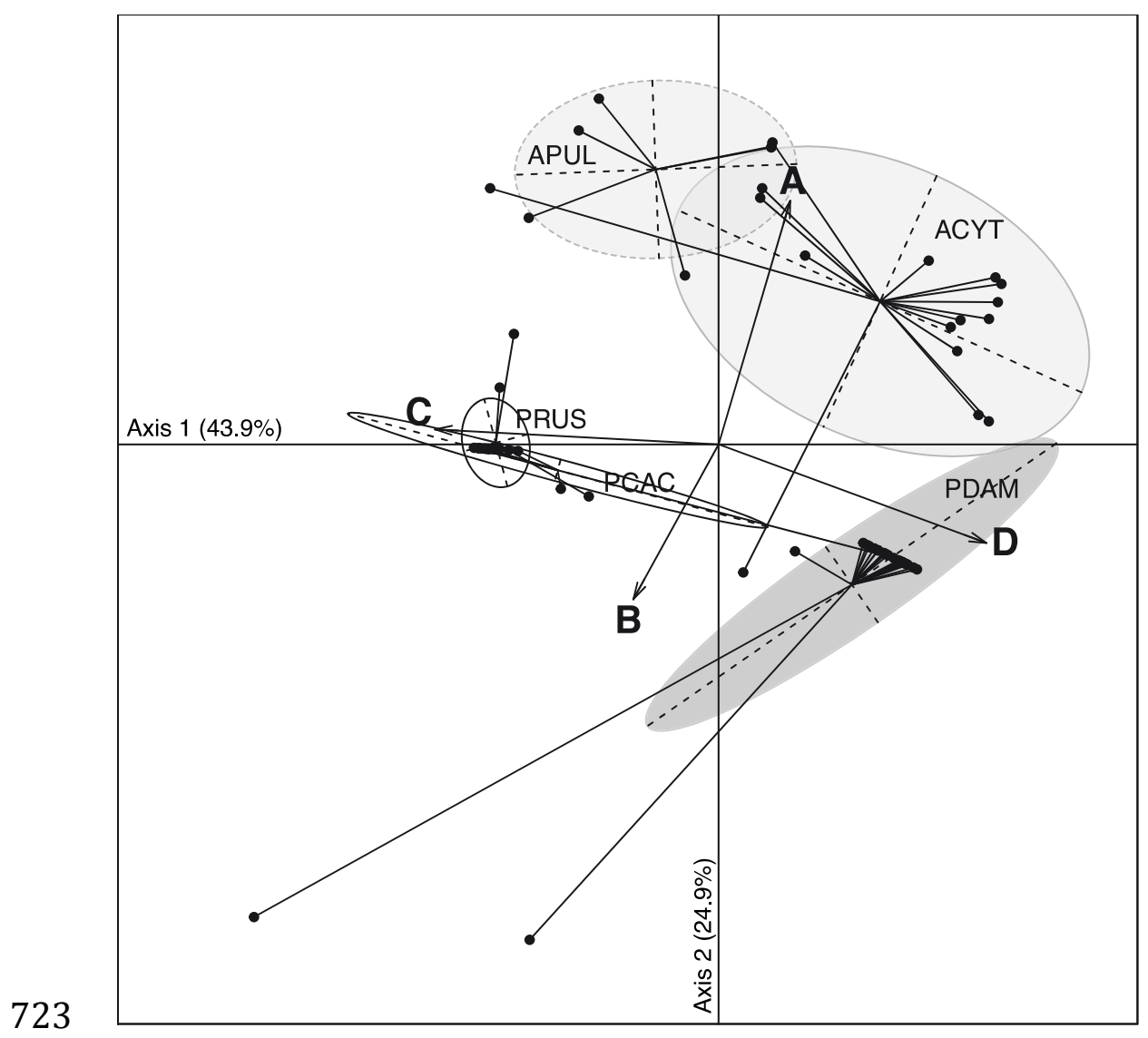

724 
725 Table 1 Comparative census of Symbiodinium clades and types associated with common coral

726 species from Moorea (A. cytherea, A. pulchra, P. damicornis, P. cactus, and P. rus) detected in a

727 previous report by Putnam et al. 2012 vs. the present study.

728

\begin{tabular}{llllll}
\hline \multirow{2}{*}{ Coral species } & \multicolumn{2}{c}{ Previous report [1] } & & \multicolumn{2}{c}{ Present study } \\
\cline { 2 - 3 } \cline { 5 - 6 } & clade(s) & type & & clades & $*$ type \\
\hline A. cytherea & $\mathrm{A}, \mathrm{D}$ & $\mathrm{A} 1, \mathrm{D} 1$ & & $\mathrm{~A}, \mathrm{C} *, \mathrm{D}$ & $\mathrm{C} 1^{*}$ \\
A. pulchra & $\mathrm{A}, \mathrm{D}$ & $\mathrm{A} 1, \mathrm{D} 1$ & & $\mathrm{~A}, \mathrm{C} *, \mathrm{D}$ & $\mathrm{ND}$ \\
P. damicornis & $\mathrm{A}, \mathrm{C}, \mathrm{D}$ & $\mathrm{DA}, \mathrm{A} 1, \mathrm{C} 15$ & & $\mathrm{~A}, \mathrm{~B} *, \mathrm{C}, \mathrm{D}$ & $\mathrm{B} 1 *$ \\
$P$. rus & $\mathrm{C}$ & $\mathrm{C} 15$ & & A*, C, D* & A13*, D1*, C15, C1* \\
P. cactus & $\mathrm{C}$ & $\mathrm{C} 1, \mathrm{C} 3, \mathrm{C} 45$ & & C, D* & C1, D1* \\
\hline
\end{tabular}

729 * novel detected Symbiodinium clade/type for the listed coral species from this study

730 ND: no data 
732 Table 2 Proportion of background clades identified within the coral hosts A. cytherea,

733 A. pulchra, P. cactus, P. damicornis and P. rus.

734

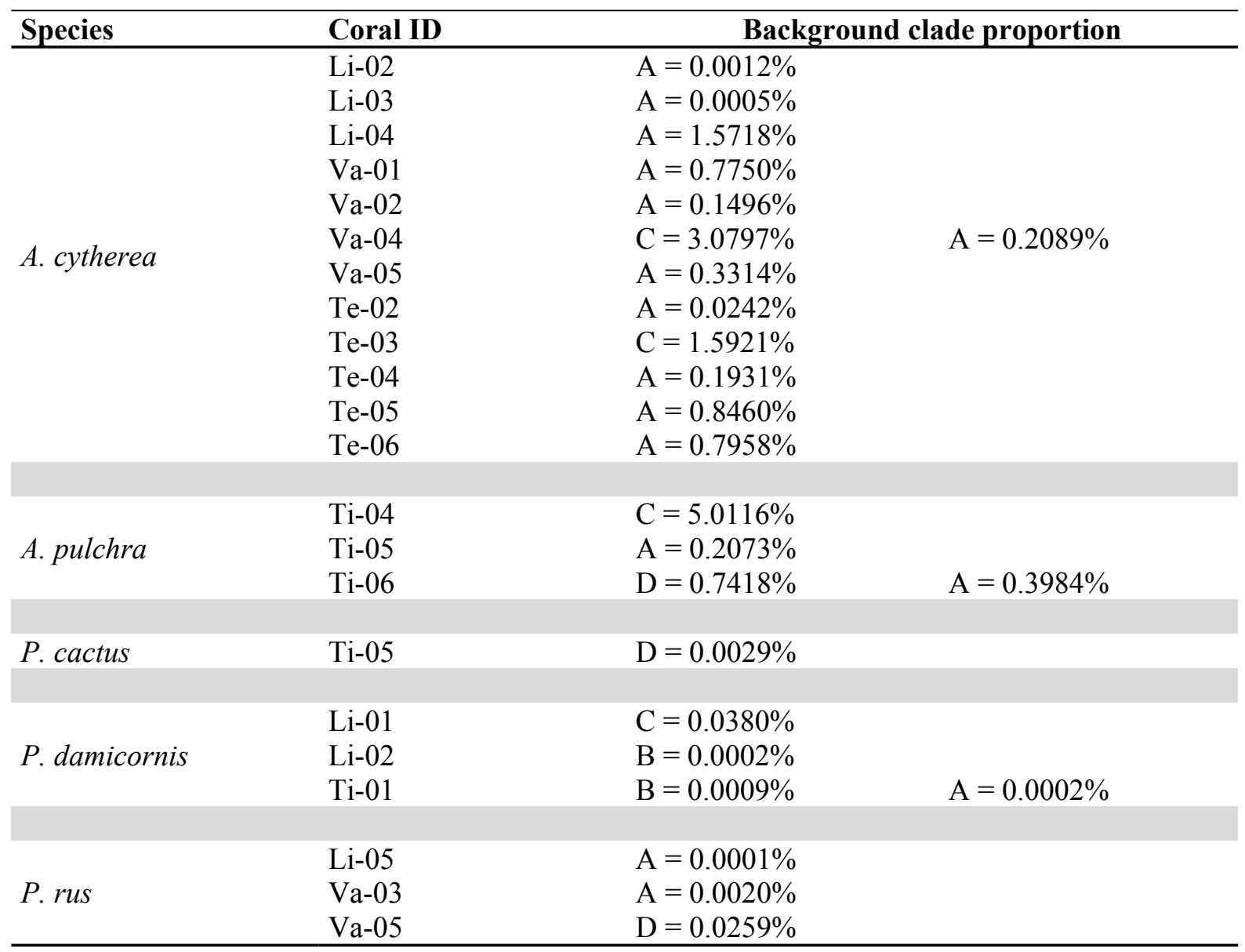

